\title{
Status of Eikonal Two-Loop Calculations with Massive Quarks
}

\author{
Nikolaos Kidonaki,1 and Philip Stephens \\ Kennesaw State University, Physics \#1202 \\ 1000 Chastain Rd., Kennesaw, GA 30144-5591
}

\begin{abstract}
We present results for two-loop diagrams with massive quarks in the eikonal approximation. Explicit expressions are given for the UV poles in dimensional regularization of several of the required integrals.
\end{abstract}

\section{Introduction}

The calculation of threshold corrections to hard scattering cross sections beyond leading logarithms requires the calculation of loop diagrams in the eikonal approximation [1]. One-loop calculations have been performed for all $2 \rightarrow 2$ partonic processes in heavy quark [2] and jet [3] production. The soft anomalous dimension matrix $\Gamma_{S}$ at one-loop allows the resummation of soft-gluon corrections at next-to-leading logarithm (NLL) accuracy [2]. The exponentiation follows from the renormalization group evolution of $\Gamma_{S}$ and involves the calculation of the ultraviolet (UV) poles in dimensional regularization of one-loop diagrams with eikonal lines. To extend resummation to next-to-next-to-leading logarithms (NNLL) two-loop calculations are required. For massless quark-antiquark scattering the two-loop $\Gamma_{S}$ was completed in [4]. For heavy quark production, however, the result is not known. In this contribution we present several results for two-loop diagrams involved in the calculation of the two-loop $\Gamma_{S}$ for massive quarks. In the eikonal approximation the usual Feynman rules are simplified by letting the
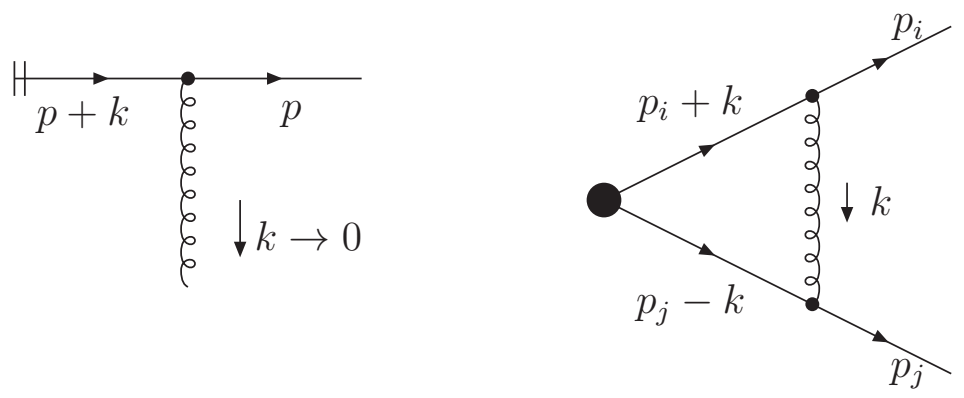

Figure 1: The eikonal approximation (left) and a one-loop diagram (right).

\footnotetext{
${ }^{1}$ Presented at DIS 2008, London, England, April 7-11, 2008
} 
gluon momentum approach zero (left diagram in Fig. 1):

$$
\bar{u}(p)\left(-i g_{s} T_{F}^{c}\right) \gamma^{\mu} \frac{i(\not p+\not d+m)}{(p+k)^{2}-m^{2}+i \epsilon} \rightarrow \bar{u}(p) g_{s} T_{F}^{c} \gamma^{\mu} \frac{\not p+m}{2 p \cdot k+i \epsilon}=\bar{u}(p) g_{s} T_{F}^{c} \frac{v^{\mu}}{v \cdot k+i \epsilon}
$$

with $p \propto v$, and $T_{F}^{c}$ the generators of $\mathrm{SU}(3)$.

\section{One-loop and two-loop diagrams}

We perform our calculation for eikonal massive quarks in Feynman gauge using dimensional regularization with $n=4-\epsilon$.

We begin with the one-loop diagram in Fig. 1. The momentum integral is given by

$$
I_{1 l}=g_{s}^{2} \int \frac{d^{n} k}{(2 \pi)^{n}} \frac{(-i) g^{\mu \nu}}{k^{2}} \frac{v_{i}^{\mu}}{v_{i} \cdot k} \frac{\left(-v_{j}^{\nu}\right)}{\left(-v_{j} \cdot k\right)} .
$$

Using Feynman parametrization, followed by integration over $k$, and after several manipulations, we find

$$
\begin{aligned}
I_{1 l}= & \frac{\alpha_{s}}{\pi}(-1)^{-1-\epsilon / 2} 2^{5 \epsilon / 2} \pi^{\epsilon / 2} \Gamma\left(1+\frac{\epsilon}{2}\right)\left(1+\beta^{2}\right) \int_{0}^{1} d x x^{-1+\epsilon}(1-x)^{-1-\epsilon} \\
& \times\left\{\int_{0}^{1} d z\left[4 z \beta^{2}(1-z)+1-\beta^{2}\right]^{-1}-\frac{\epsilon}{2} \int_{0}^{1} d z \frac{\ln \left[4 z \beta^{2}(1-z)+1-\beta^{2}\right]}{4 z \beta^{2}(1-z)+1-\beta^{2}}+\mathcal{O}\left(\epsilon^{2}\right)\right\}
\end{aligned}
$$

where $\beta=\sqrt{1-4 m^{2} / s}$. The integral over $x$ contains both UV and infrared (IR) singularities. We isolate the UV singularities, $\int_{0}^{1} d x x^{-1+\epsilon}(1-x)^{-1-\epsilon}=\frac{1}{\epsilon}+\mathrm{IR}$, and find the UV pole and constant terms at one loop:

$$
\begin{aligned}
I_{1 l}^{U V}=\frac{\alpha_{s}}{\pi} \frac{\left(1+\beta^{2}\right)}{2 \beta}\{ & \frac{1}{\epsilon} \ln \left(\frac{1-\beta}{1+\beta}\right)+\frac{1}{2}\left(4 \ln 2+\ln \pi-\gamma_{E}-i \pi\right) \ln \left(\frac{1-\beta}{1+\beta}\right) \\
& \left.+\frac{1}{4} \ln ^{2}(1+\beta)-\frac{1}{4} \ln ^{2}(1-\beta)-\frac{1}{2} \operatorname{Li}_{2}\left(\frac{1+\beta}{2}\right)+\frac{1}{2} \operatorname{Li}_{2}\left(\frac{1-\beta}{2}\right)\right\} .
\end{aligned}
$$

More details on this one-loop integral are given in [5]. We now continue with the two-loop diagrams (these are the eikonal versions of the diagrams involved in the calculation of the twoloop heavy quark form factor [6]). In Fig. 2, we show a diagram with two gluons exchanged between the massive quarks (left) and the crossed diagram (right). We denote by $I_{1}$ the integral for the first diagram and by $I_{2}$ that for the crossed diagram. We have

$$
I_{1}=g_{s}^{4} \int \frac{d^{n} k_{1}}{(2 \pi)^{n}} \frac{d^{n} k_{2}}{(2 \pi)^{n}} \frac{(-i) g^{\mu \nu}}{k_{1}^{2}} \frac{(-i) g^{\rho \sigma}}{k_{2}^{2}} \frac{v_{i}^{\mu}}{v_{i} \cdot k_{1}} \frac{v_{i}^{\rho}}{v_{i} \cdot\left(k_{1}+k_{2}\right)} \frac{\left(-v_{j}^{\nu}\right)}{-v_{j} \cdot k_{1}} \frac{\left(-v_{j}^{\sigma}\right)}{-v_{j} \cdot\left(k_{1}+k_{2}\right)} .
$$

We note that $I_{1}$ is symmetric under $k_{1} \leftrightarrow k_{2}$ as is the integral for the crossed diagram, $I_{2}$. Utilizing the properties of these two integrals and the one-loop integral, $I_{1 l}$, we find the relation

$$
I_{1}=\frac{1}{2}\left(I_{1 l}\right)^{2}-I_{2}
$$



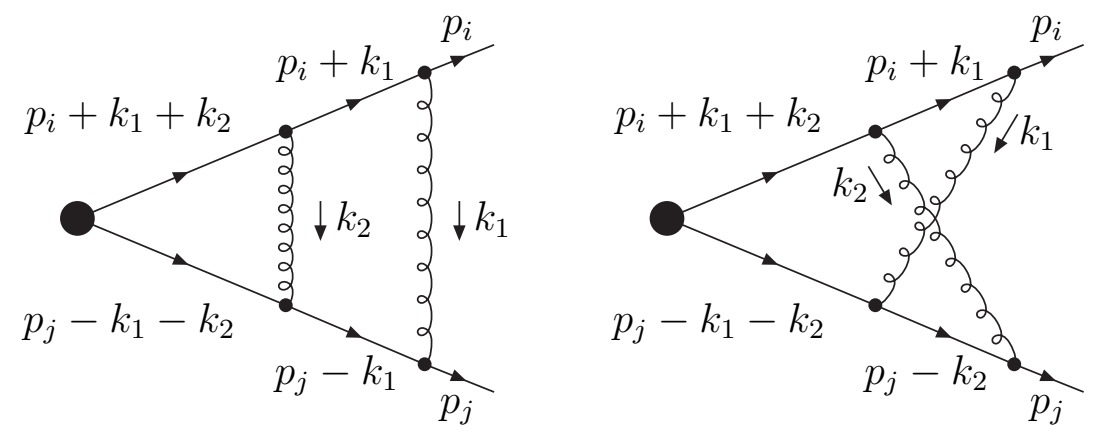

Figure 2: Two loop diagrams with two-gluon exchanges.

Therefore $I_{1}$ is determined once we calculate $I_{2}$. For the crossed diagram, we have

$$
I_{2}=g_{s}^{4} \int \frac{d^{n} k_{1}}{(2 \pi)^{n}} \frac{d^{n} k_{2}}{(2 \pi)^{n}} \frac{(-i) g^{\mu \nu}}{k_{1}^{2}} \frac{(-i) g^{\rho \sigma}}{k_{2}^{2}} \frac{v_{i}^{\mu}}{v_{i} \cdot k_{1}} \frac{v_{i}^{\rho}}{v_{i} \cdot\left(k_{1}+k_{2}\right)} \frac{\left(-v_{j}^{\nu}\right)}{-v_{j} \cdot\left(k_{1}+k_{2}\right)} \frac{\left(-v_{j}^{\sigma}\right)}{-v_{j} \cdot k_{2}} .
$$

We can set $\epsilon=0$ in the $k_{2}$ integral since it is UV finite. Then

$$
\begin{aligned}
I_{2}= & -i \frac{\alpha_{s}^{2}}{\pi^{2}} 2^{-4} \pi^{-2}\left(1+\beta^{2}\right)^{2} \int_{0}^{1} d z \int_{0}^{1} \frac{d y}{2 \beta^{2}(1-y)^{2} z^{2}-2 \beta^{2}(1-y) z-\frac{\left(1-\beta^{2}\right)}{2}} \\
& \times \int \frac{d^{n} k_{1}}{k_{1}^{2} v_{i} \cdot k_{1}\left[\left(v_{i}-v_{j}\right) z+v_{j}\right] \cdot k_{1}} .
\end{aligned}
$$

Now we proceed with the $k_{1}$ integral and separate the UV and IR poles. After many steps, we find the $1 / \epsilon^{2}$ and $1 / \epsilon \mathrm{UV}$ poles of $I_{2}$ :

$$
\begin{aligned}
& I_{2}^{U V}=-\frac{\alpha_{s}^{2}}{\pi^{2}} \frac{\left(1+\beta^{2}\right)^{2}}{4 \beta^{2}} \frac{1}{\epsilon}\left\{\operatorname { l n } ( \frac { 1 - \beta } { 1 + \beta } ) \left[2 \operatorname{Li}_{2}\left(\frac{2 \beta}{1+\beta}\right)+4 \operatorname{Li}_{2}\left(\frac{1-\beta}{1+\beta}\right)\right.\right. \\
&\left.+2 \operatorname{Li}_{2}\left(\frac{-(1-\beta)}{1+\beta}\right)-\ln (1+\beta) \ln (1-\beta)-\zeta_{2}\right] \\
&-2 \ln ^{2}\left(\frac{1-\beta}{1+\beta}\right) \ln \left(\frac{1+\beta}{2 \beta}\right)+\left.\frac{1}{3} \ln ^{3}(1-\beta)-\frac{1}{3} \ln ^{3}(1+\beta)-\operatorname{Li}_{3}\left(\frac{(1-\beta)^{2}}{(1+\beta)^{2}}\right)+\zeta_{3}\right\} .
\end{aligned}
$$

We now proceed with the diagrams in Fig. 3 that involve internal quark and gluon loops. For the quark loop we find

$$
I_{q l}=(-1) n_{f} g_{s}^{4} \int \frac{d^{n} k}{(2 \pi)^{n}} \frac{d^{n} l}{(2 \pi)^{n}} \frac{v_{i}^{\mu}}{v_{i} \cdot k} \frac{\left(-v_{j}^{\rho}\right)}{\left(-v_{j} \cdot k\right)} \frac{(-i) g^{\mu \nu}}{k^{2}} \frac{(-i) g^{\rho \sigma}}{k^{2}} \operatorname{Tr}\left[-i \gamma^{\nu} \frac{i \not l}{l^{2}}(-i) \gamma^{\sigma} i \frac{(\not-\not l)}{(l-k)^{2}}\right] .
$$

After many steps (see also [5]) we extract the UV poles

$$
\begin{aligned}
I_{q l}^{U V}=-n_{f} & \frac{\alpha_{s}^{2}}{\pi^{2}} \frac{\left(1+\beta^{2}\right)}{6 \beta}\left\{\frac{1}{\epsilon^{2}} \ln \left(\frac{1-\beta}{1+\beta}\right)+\frac{1}{\epsilon}\left[-\operatorname{Li}_{2}\left(\frac{1+\beta}{2}\right)+\operatorname{Li}_{2}\left(\frac{1-\beta}{2}\right)\right.\right. \\
& \left.\left.+\frac{1}{2} \ln ^{2}(1+\beta)-\frac{1}{2} \ln ^{2}(1-\beta)+\left(\frac{7}{12}+4 \ln 2+\ln \pi-\gamma_{E}-i \pi\right) \ln \left(\frac{1-\beta}{1+\beta}\right)\right]\right\} .
\end{aligned}
$$



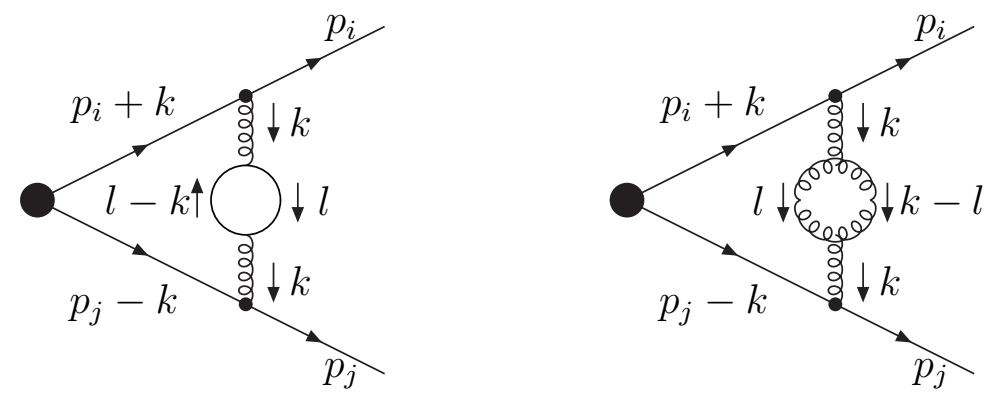

Figure 3: Two-loop diagrams with quark and gluon loops.

For the gluon-loop integral, we have

$$
\begin{aligned}
I_{g l}= & \frac{1}{2} g_{s}^{4} \int \frac{d^{n} k}{(2 \pi)^{n}} \frac{d^{n} l}{(2 \pi)^{n}} \frac{v_{i}^{\mu}}{v_{i} \cdot k} \frac{\left(-v_{j}^{\nu}\right)}{\left(-v_{j} \cdot k\right)} \frac{(-i) g^{\mu \mu^{\prime}}}{k^{2}} \frac{(-i) g^{\rho \rho^{\prime}}}{l^{2}} \frac{(-i) g^{\sigma \sigma^{\prime}}}{(k-l)^{2}} \frac{(-i) g^{\nu \nu^{\prime}}}{k^{2}} \\
& \times\left[g^{\mu^{\prime} \rho}(k+l)^{\sigma}+g^{\rho \sigma}(k-2 l)^{\mu^{\prime}}+g^{\sigma \mu^{\prime}}(-2 k+l)^{\rho}\right] \\
& \times\left[g^{\rho^{\prime} \nu^{\prime}}(l+k)^{\sigma^{\prime}}+g^{\nu^{\prime} \sigma^{\prime}}(-2 k+l)^{\rho^{\prime}}+g^{\sigma^{\prime} \rho^{\prime}}(k-2 l)^{\nu^{\prime}}\right] .
\end{aligned}
$$

We calculate the UV poles and find

$$
\begin{aligned}
I_{g l}^{U V}=-\frac{19}{96} & \frac{\alpha_{s}^{2}}{\pi^{2}} \frac{\left(1+\beta^{2}\right)}{\beta}\left\{\frac{1}{\epsilon^{2}} \ln \left(\frac{1-\beta}{1+\beta}\right)+\frac{1}{\epsilon}\left[-\operatorname{Li}_{2}\left(\frac{1+\beta}{2}\right)+\operatorname{Li}_{2}\left(\frac{1-\beta}{2}\right)\right.\right. \\
& \left.\left.+\frac{1}{2} \ln ^{2}(1+\beta)-\frac{1}{2} \ln ^{2}(1-\beta)+\left(\frac{58}{57}+4 \ln 2+\ln \pi-\gamma_{E}-i \pi\right) \ln \left(\frac{1-\beta}{1+\beta}\right)\right]\right\} .
\end{aligned}
$$

We also have to add a diagram to those in Fig. 3 involving a ghost loop. The corresponding integral is

$$
I_{g h}=(-1) g_{s}^{4} \int \frac{d^{n} k}{(2 \pi)^{n}} \frac{d^{n} l}{(2 \pi)^{n}} \frac{v_{i}^{\mu}}{v_{i} \cdot k} \frac{\left(-v_{j}^{\rho}\right)}{\left(-v_{j} \cdot k\right)} \frac{i}{l^{2}} l^{\nu} \frac{i}{(l-k)^{2}}(l-k)^{\sigma} \frac{(-i) g^{\mu \nu}}{k^{2}} \frac{(-i) g^{\rho \sigma}}{k^{2}}
$$

and a calculation of its UV poles gives

$$
\begin{aligned}
I_{g h}^{U V}=-\frac{\alpha_{s}^{2}}{\pi^{2}} & \frac{\left(1+\beta^{2}\right)}{96 \beta}\left\{\frac{1}{\epsilon^{2}} \ln \left(\frac{1-\beta}{1+\beta}\right)+\frac{1}{\epsilon}\left[-\operatorname{Li}_{2}\left(\frac{1+\beta}{2}\right)+\operatorname{Li}_{2}\left(\frac{1-\beta}{2}\right)\right.\right. \\
& \left.\left.+\frac{1}{2} \ln ^{2}(1+\beta)-\frac{1}{2} \ln ^{2}(1-\beta)+\left(\frac{4}{3}+4 \ln 2+\ln \pi-\gamma_{E}-i \pi\right) \ln \left(\frac{1-\beta}{1+\beta}\right)\right]\right\} .
\end{aligned}
$$

We also note that the integral for another diagram involving an internal gluon loop with a four-gluon vertex vanishes.

There are additional diagrams not discussed here, also including self-energies and counterterms. The color factors for all diagrams have been calculated and must be accounted for in the final result. 


\section{Acknowledgements}

The work of N.K. was supported by the National Science Foundation under Grant No. PHY 0555372 .

\section{References}

[1] Slides:

http://indico.cern.ch/contributionDisplay.py?contribId=200

\&sessionId $=13 \&$ confId $=24657$

[2] N. Kidonakis and G. Sterman, Phys. Lett. B387, 867 (1996); Nucl. Phys. B505 321 (1997) hep-ph/9705234].

[3] N. Kidonakis, G. Oderda and G. Sterman, Nucl. Phys. B531, 365 (1998) hep-ph/9803241.

[4] S.M. Aybat, L.J. Dixon and G. Sterman, Phys. Rev. Lett. 97, 072001 (2006) hep-ph/0606254; Phys. Rev. D74, 074004 (2006) hep-ph/0607309.

[5] N. Kidonakis, A. Sabio Vera and P. Stephens, arXiv:0802.4240 [hep-ph] (2008).

[6] W. Bernreuther, R. Bonciani, T. Gehrmann, R. Heinesch, T. Leineweber, P. Mastrolia and E. Remiddi, Nucl. Phys. B706, 245 (2005) [hep-ph/0406046]. 